\title{
Finite Element Simulation of Stress Evolution in a Frictional Contact System
}

\author{
H.L. Xing ${ }^{1}$, P. Mora ${ }^{1}$, and A. Makinouchi ${ }^{2}$ \\ ${ }^{1}$ QUAKES, The University of Queensland, St. Lucia, Brisbane, QLD 4072, Australia \\ \{xing, mora\} @quakes . uq. edu . au \\ ${ }^{2}$ Integrated V-CAD Research Program \\ The Institute of Physical and Chemical Research, Japan
}

\begin{abstract}
A 3-dimensional finite element algorithm for modeling nonlinear frictional contact behaviours between deformable bodies with the node-to-point contact element strategy has been proposed and applied here to investigate stress evolution processes of a nonlinear frictional contact system. The numerical results of a typical intra-plate fault bend model demonstrate the efficiency and usefulness of this algorithm.
\end{abstract}

\section{Introduction}

The understanding, simulation and prediction of behaviors of frictional contact systems are very important in both theory and practical applications. Although the important progresses have been achieved in the computational mechanics, how to describe the complex phenomena in a frictional contact system with reasonable accuracy still remains a problem to be overcome. Up to now, it is difficult for most of the existing finite element codes to get a stable solution even with the assumption that the interface parameters are constant during the frictional process. An arbitrarily shaped contact element strategy, named as node-to-point contact element strategy, was proposed based on the static-explicit algorithm and applied to handle the frictional contact between deformable bodies with stick and finite frictional slip [1-4]. This paper will focus on how to extend the algorithm to the simulation of stress evolution phenomena in a frictional contact system.

\section{Finite Element Formulation}

The updated Lagrangian rate formulation is employed to describe the non-linear contact problem. The rate type equilibrium equation and the boundary at the current configuration are equivalently expressed by a principle of virtual velocity of the form

$\int_{V}\left(\sigma_{i j}^{J}-D_{i k} \sigma_{k j}+\sigma_{i k} L_{j k}-\sigma_{i k} D_{k j}\right) \delta L_{i j} d V=\int_{S_{F}} \dot{F}_{i} \delta v_{i} d S+\int_{S_{c}^{1}} \dot{f}_{i}^{l} \delta v_{i} d S+\int_{S_{c}^{2}} \dot{f}_{i}^{2} \delta v_{i} d S(1)$ 
where $V$ and $S$ denote respectively the domains occupied by the total deformable body $B$ and its boundary at time t; $S_{F}$ is a part of the boundary of $S$ on which the rate of traction $\dot{F}_{i}$ is prescribed; $\delta \boldsymbol{v}$ is the virtual velocity field which satisfies the condition $\boldsymbol{\delta} \boldsymbol{v}=\boldsymbol{0}$ on the velocity boundary; $\sigma_{i j}^{J}$ is the Jaumann rate of Cauchy stress $\sigma_{i j} ; \boldsymbol{L}$ is the velocity gradient tensor, $\boldsymbol{L}=\partial v / \partial \boldsymbol{x} ; \boldsymbol{D}$ is the symmetric part of $\boldsymbol{L} ; \boldsymbol{f}^{\alpha}$ is the rate of contact stress on contact interface $S_{c}^{\alpha}$ of the body $\alpha$ and calculated as follows.

Friction is by nature a path-dependent dissipative phenomenon that requires the integration along the slip path. In this study, a standard Coulomb friction model is applied in an analogous way to the flow plasticity rule, which governs the slipping behaviour. The basic formulations are summarized below [1]-[3] (The tiled ( ) above a variable denotes a relative component between slave and master bodies, and $l, m=1,2$; $i, j, k=1,3$ respectively in this paper).

Based on experimental observations, an increment decomposition is assumed

$$
\Delta \tilde{u}_{m}=\Delta \tilde{u}_{m}^{e}+\Delta \tilde{u}_{m}^{p}
$$

where $\Delta \tilde{u}_{m}^{e}$ and $\Delta \tilde{u}_{m}^{p}$ respectively represent the stick (reversible) and the slip (irreversible) part of $\Delta \tilde{u}_{m}$, the relative displacement increment component along direction $m$ on the contact interface. The slip is governed by the yield condition

$$
F=\sqrt{f_{m} f_{m}}-\bar{F},
$$

where $f_{m}(m=1,2)$ is the frictional stress component along the tangential direction $m$; $\bar{F}$, the critical frictional stress, $\bar{F}=\mu f_{n} ; \mu$ is the friction coefficient, it may depend on the normal contact pressure $f_{n}$, the equivalent slip velocity $\dot{\tilde{u}}_{e q}\left(=\sqrt{\dot{\tilde{u}}_{m} \dot{\tilde{u}}_{m}}\right)$ and the state variable $\varphi$, i.e. $\mu=\mu\left(f_{n}, \dot{\tilde{u}}_{e q}, \varphi\right)$ (e.g. [5]).

If $F<0$, contact is in the sticking state and treated as a linear elasticity, i.e.

$$
f_{m}=E_{t} \tilde{u}_{m}^{e}=E_{t} \sum \Delta \tilde{u}_{m}^{e},
$$

where $E_{t}$ is a constant in the tangential direction.

When $F=0$, the friction changes its character from the stick to the slip. The frictional stress can be described as

$$
f_{m}=\eta_{m} \bar{F} \text { and } \eta_{m}=f_{m}^{e} / \sqrt{f_{l}^{e} f_{l}^{e}}
$$

where $f_{m}^{e}=E_{t}\left(\tilde{u}_{m}-\left.\tilde{u}_{m}^{p}\right|_{0}\right)$, and $\left.\tilde{u}_{m}^{p}\right|_{0}$ is the value of $\tilde{u}_{m}^{p}$ at the beginning of this step. 
The linearized form of Eq. (5) can be rewritten as

$$
d f_{l}=\frac{\bar{F} E_{t}}{\sqrt{f_{m}^{e} f_{m}^{e}}}\left(\delta_{l m}-\eta_{l} \eta_{m}\right) d \tilde{u}_{m}+\eta_{l} \mu\left(d f_{n}+\frac{\partial \mu}{\partial f_{n}} d f_{n}\right)+\eta_{l} f_{n}\left(\frac{\partial \mu}{\partial \dot{\tilde{u}}_{e q}} d \dot{\tilde{u}}_{e q}+\frac{\partial \mu}{\partial \varphi} d \varphi\right)
$$

In addition, the penalty parameter method is chosen to satisfy the normal impenetrability condition. Finally, the frictional contact stress acting on a slave node can be described as (denote $\dot{f}_{3}=\dot{f}_{n}$ )

$$
\dot{f}_{i}=G_{i j} \dot{\tilde{u}}_{j}+\dot{f}_{\varphi i}
$$

where $\boldsymbol{G}$ is the frictional contact matrix; $\dot{f}_{\varphi i}$ is from the contribution of the terms related with $\varphi$.

A node-to-point contact element strategy was proposed to handle the frictional contact problems between deformable bodies [1]-[4]. Assume a slave node $s$ has contacted with point $c$ on a surface element (master segment) $E^{\prime}$, and the surface element $E^{\prime}$ consists of $\gamma$ nodes ( $\gamma=4$ in this paper if without special notation), thus the term related with contact in Eq. (1) can be described as $(\alpha=1,(\gamma+1), \beta=1,(\gamma+1))$

$$
\dot{f}_{i}\left(\delta u_{s i}-\delta u_{c i}\right)=\delta \tilde{u}_{s c i \beta}\left(\left[\bar{K}_{f i k}\right]_{\beta \alpha} \dot{u}_{s c k \alpha}+R_{\beta} \dot{f}_{\varphi i}\right)
$$

where

$$
\left\lfloor\bar{K}_{f i k}\right\rfloor_{\beta \alpha}=R_{\beta} \boldsymbol{e}_{i} \cdot\left\{G_{h k} R_{\alpha} \boldsymbol{e}_{h}+\left(H_{j m} \hat{\boldsymbol{e}}_{j}\left(\left(\bar{C}_{l l} R_{\alpha, m}-\bar{C}_{m l} R_{\alpha, l}\right) \boldsymbol{e}_{k} \cdot \tilde{\boldsymbol{x}}+R_{\alpha}\left(\bar{C}_{l l} \hat{\boldsymbol{e}}_{m}-\bar{C}_{m l} \hat{\boldsymbol{e}}_{l}\right) \cdot \boldsymbol{e}_{k}\right)\right)\right\}
$$

$$
(h=1,3, l \neq m \text { and no sum on } l) \text {, }
$$

here $\bar{C}_{m l}=C_{m l}-g_{n} \boldsymbol{n} \cdot \hat{\boldsymbol{e}}_{m, l}, C_{m l}=\hat{\boldsymbol{e}}_{m} \cdot \hat{\boldsymbol{e}}_{l}, \wp=\bar{C}_{11} \bar{C}_{22}-\bar{C}_{12} \bar{C}_{21}, \quad \tilde{\boldsymbol{x}}=\boldsymbol{x}_{s}-\boldsymbol{x}_{c}$, $E_{i j m}=\hat{\boldsymbol{e}}_{i, m} \cdot \hat{\boldsymbol{e}}_{j} \quad, \quad H_{j m}=\hat{f}_{i} E_{i j m} / \wp \quad, \quad \dot{\boldsymbol{u}}_{s c}=\left[\begin{array}{lllll}\dot{\boldsymbol{u}}_{s} & \dot{\boldsymbol{u}}_{1} & \dot{\boldsymbol{u}}_{2} & \ldots & \dot{\boldsymbol{u}}_{\gamma}\end{array}\right]$, , $\boldsymbol{R}=\left[\begin{array}{lllll}1 & -N_{1} & -N_{2} & \ldots & -N_{\gamma}\end{array}\right]^{T}, N_{p}(p=1, \gamma)$ is the shape function value of the point $c$ on the surface element $E^{\prime}, \hat{\boldsymbol{e}}_{i}$ and $\boldsymbol{e}_{i}$ are respectively the base vectors of the local natural and the local Cartesian coordinate systems on the master segment; $g_{n}=\boldsymbol{n} \cdot\left(\boldsymbol{x}_{s}-\boldsymbol{x}_{c}\right)$, while $\boldsymbol{x}_{s}$ and $\boldsymbol{x}_{c}$ are the position coordinates of a slave node and its corresponding contact point on a master surface, respectively.

The explicit time integration algorithm is applied with the $R$-minimum to limit the step size for avoiding a drastic change in states within an incremental step [e.g. 4]. In combination with the above equations, Eq. (1) can be rewritten as

$$
\left(\boldsymbol{K}+\boldsymbol{K}_{f}\right) \Delta \boldsymbol{u}=\Delta \boldsymbol{F}+\Delta \boldsymbol{F}_{f}
$$


here $\boldsymbol{K}$ is the standard stiffness matrix corresponding to the total body $B ; \Delta \boldsymbol{F}$ is the external force increment subjected to body $B$ on $S_{F} ; \Delta \boldsymbol{u}$ is the nodal displacement increment; $\boldsymbol{K}_{f}$ and $\Delta \boldsymbol{F}_{f}$ are the stiffness matrices and the force increments of all the node-to-point contact elements.

\section{Numerical Investigation of Stress Evolution}

A typical intra-plate fault bend model $\left(300 \times 300 \times 50 \mathrm{~mm}^{3}\right)$ is analyzed here, which has a pre-cut fault that is artificially bent by an angle of $5.6^{\circ}$ at the center $E$ of the fault. The details of the geometry, the boundary conditions are shown in Figure 1. There exists no relative motion along the interface at the both ends (as depicted using the thick black lines CD and FG in Fig. 1). This can be easily achieved using the 'stick' algorithm in the code. While, for the other part of the fault interface (i.e. segments DE and EF in Figure 1), the widely applied rate- and state-dependent friction law [5] is used here with the following parameters: $\mu=0.60+(0.010-0.025) \ln (V / 0.001), d \varphi / d t=0$. Thus, the total fault consists of four fault segments: $\mathrm{CD}, \mathrm{DE}, \mathrm{EF}$ and FG in Fig. 1. Here all the materials have the same properties: density $\rho=2.60 \mathrm{~g} / \mathrm{cm}^{3}$, Young's modulus $E=44.8 \mathrm{GPa}$ and Poisson's ratio $\gamma=0.12$. As for the loading conditions, two loading stages are applied here: firstly, the pressure on the surfaces A and B are loaded up to $10 \mathrm{MPa}$, then sustaining this pressure on surface $\mathrm{A}$, while all the nodes on surface B are moved in the $\mathrm{x}$-direction at the velocity $V x=-0.001 \mathrm{~mm} / \mathrm{sec}$.

The stress evolution of the total system during the loading processes is calculated and some results at the different loading time are shown in Figs. 2 and 3. At the beginning of the second loading stage (i.e. at the end of the first loading stage), the stress distribution is rather smooth except that at the both ends as shown in Fig. 2, and the same situation remains until the nodes start to change their stick states to the slip (see Figs. 3: time=228.7s). However, with the increase of the prescribed displacement, the stress on the segment DE begins to decrease and to redistribute locally due to the local energy release induced by the state changes from the stick to the slip on the segment DE. This also results in the stress increase for the nodes around the segments CD and EF (see Fig. 3: Time=292.75s - 296.15s). Afterwards, the stress increases much for most of the nodes except the local energy release zone around the segment DE (see, e.g. Fig. 3: Time=302.98s). With the transition of the stick-slip instability and the occurrence of energy release at the node on the fault segment EF, the stress deceases and redistributes locally for the corresponding nodes which have just entered the slip state, while the stress of neighboring node that is in the stick state increases (see, e.g. Figs. 3: Time $=311.08 \mathrm{~s}-369.78 \mathrm{~s}$ ). Finally, this also causes the obvious stress increase at both end zones (i.e. segments CD and FG in Fig. 1) (as shown in Figs. 3: Time=329.93s -369.78 s). 


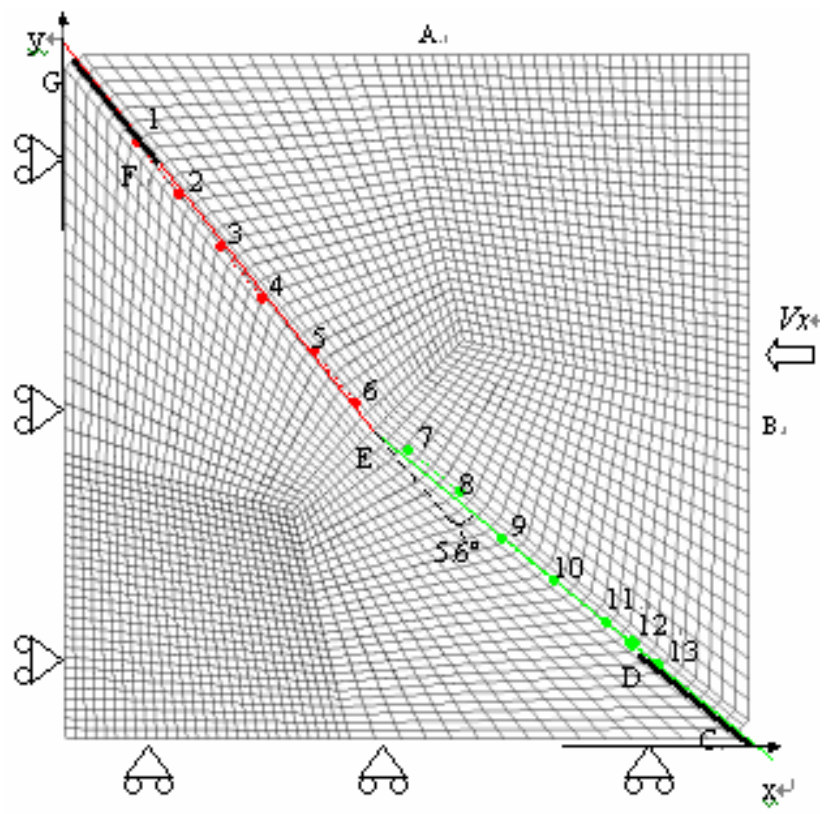

Fig. 1. The mesh, the boundary conditions and prescribed nodal positions used for the fault bend model in the $\mathrm{x}-\mathrm{y}$ cross section. Here all the nodes at the surface marked by a triangular with two circles $(\widehat{O})$ are free along the direction of two circles but fixed at the other direction
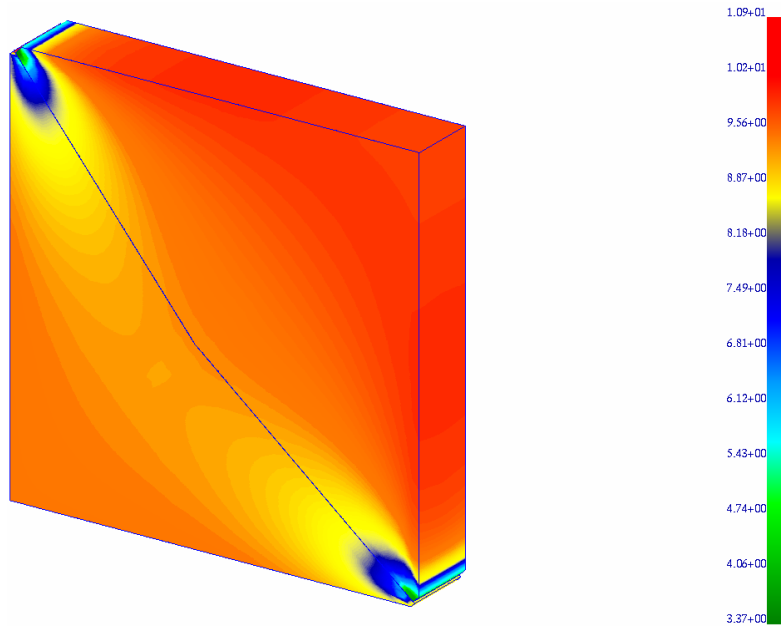

Fig. 2. Mises equivalent stress $\bar{\sigma}$ distribution at the end of the first loading stage(here $\bar{\sigma}=\sqrt{3 \sigma_{i j}^{\prime} \sigma_{i j}^{\prime} / 2}, \sigma_{i j}^{\prime}$ is the deviator stress tensor, unit: $\left.\mathrm{MPa}\right)$ 

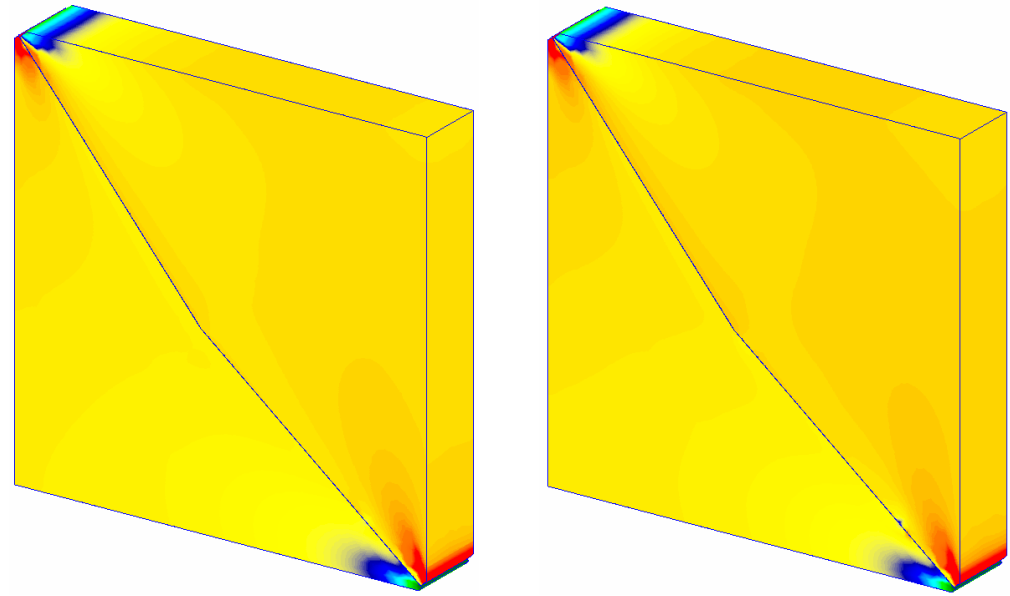

Time (s): $\quad 228.7$

292.75
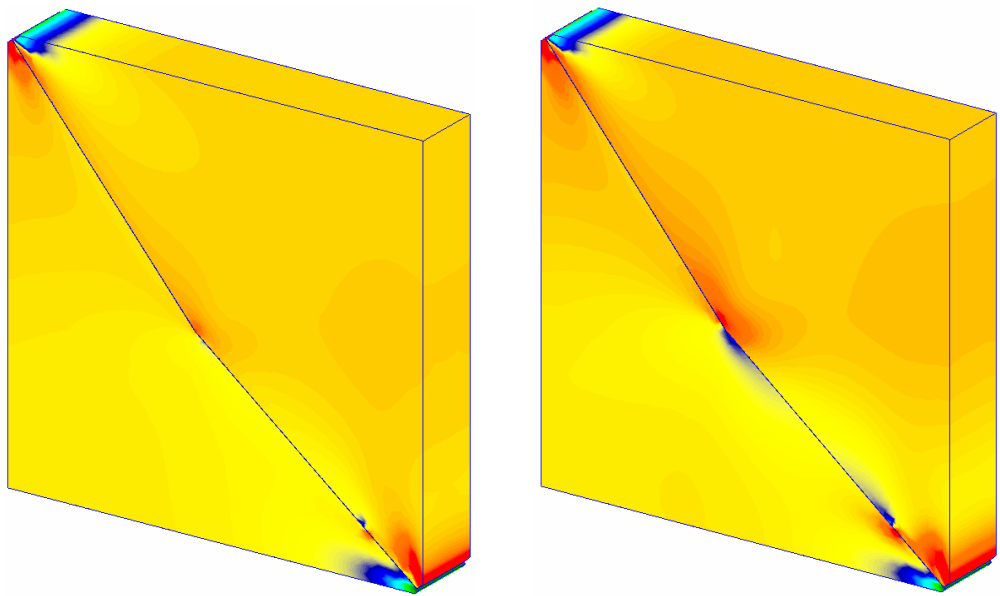

Time (s):

$$
293.44
$$

296.15

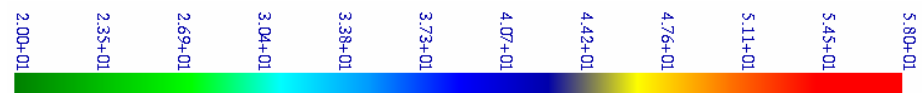

Fig. 3. Mises equivalent stress $\bar{\sigma}$ variation during the second loading stage (here $\bar{\sigma}=\sqrt{3 \sigma_{i j}^{\prime} \sigma_{i j}^{\prime} / 2}, \sigma_{i j}^{\prime}$ is the deviator stress tensor, unit: $\left.\mathrm{MPa}\right)$ 

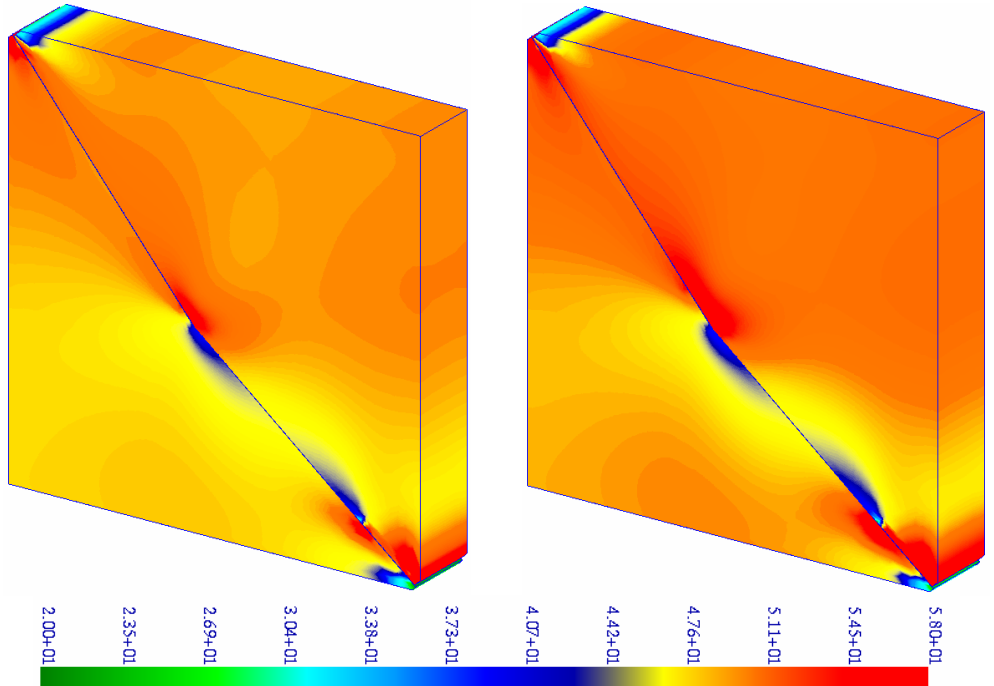

Time (s):

302.98

311.08
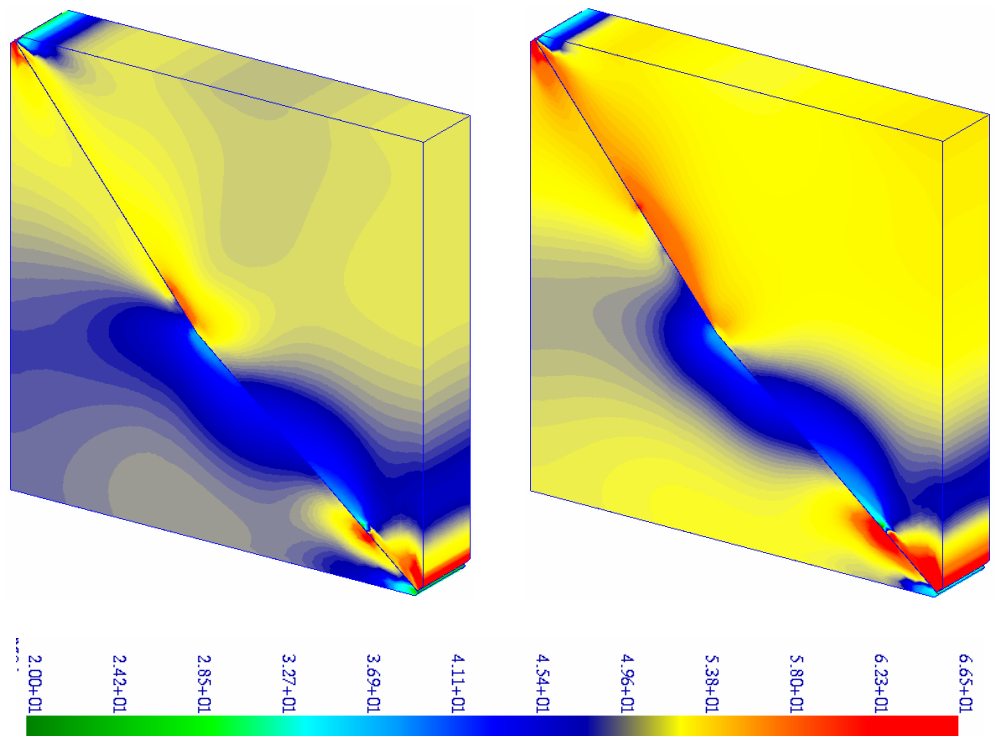

Time (s): $\quad 313.41$

329.93

Fig. 3. (continued) 

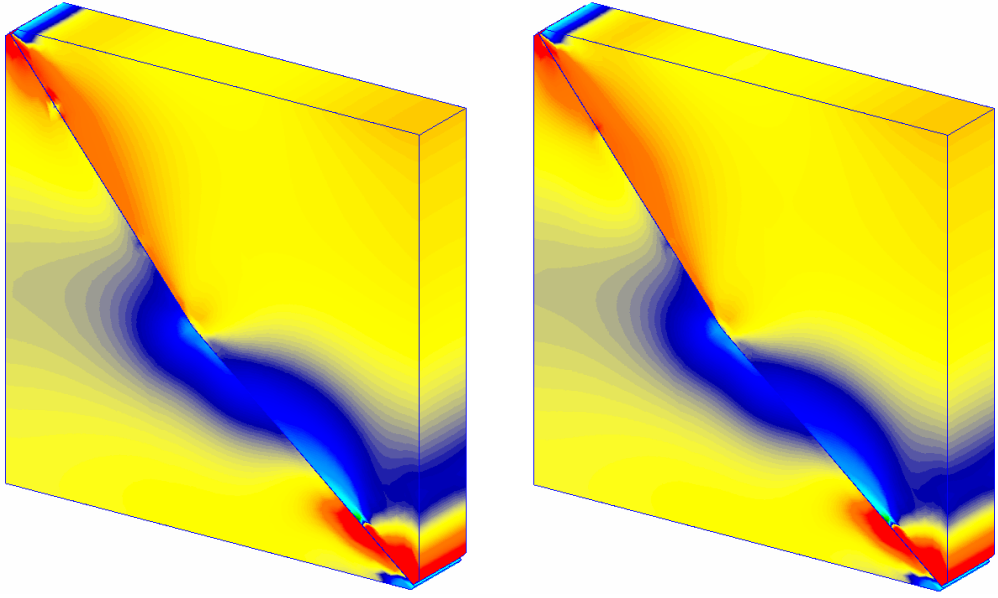

Time (s):

335.19

335.42
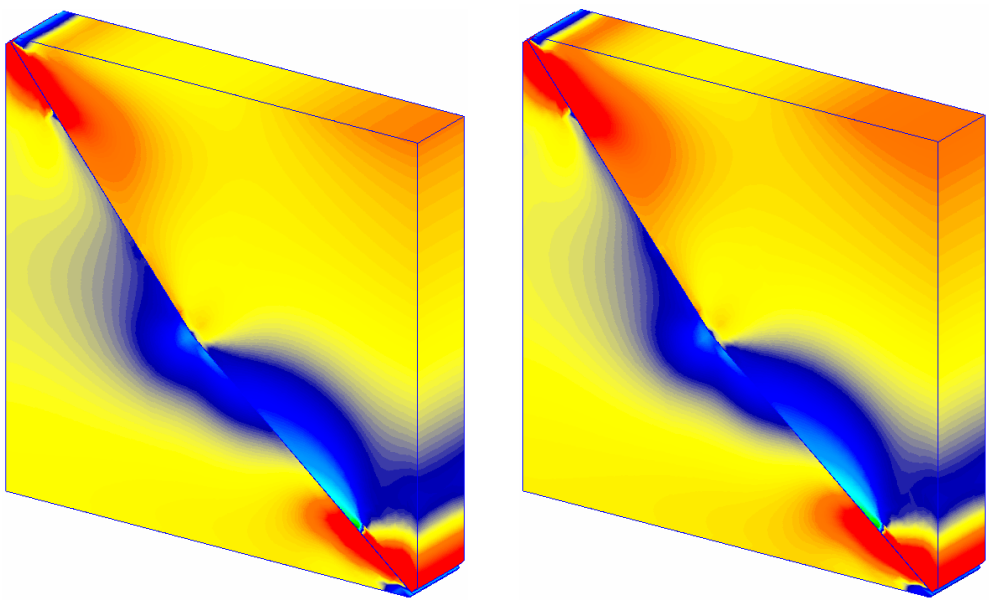

Time (s):

346.08

369.78

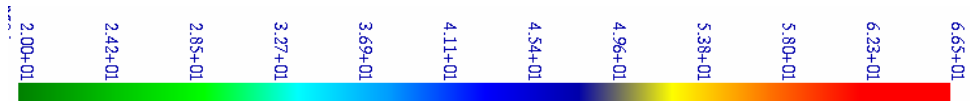

Fig. 3. (continued) 


\section{Discussion and Conclusions}

A 3-dimensional finite element code for modeling nonlinear frictional contact behaviours between deformable bodies with the node-to-point contact element strategy has been developed and applied here to investigate stress evolution processes of a typical intra-plate fault system. The above numerical results show that: (1) During the nucleation process of the stick-slip instability, the stress distribution is rather smooth except that around both ends; (2) Once a stick node enters the slip, the stress decreases and redistributes locally at the corresponding slip zone, but increases in a local narrow zone around its neighbour nodes being in the stick. Thus these stick nodes are 'pushed' to the slip state by the neighbour nodes entering the slip. (3). Comparison with the calculation results for a flat fault [2], the fault bend has significant influence on the nucleation, termination and restart of the stick-slip instability along the intra-plate fault, and further on the corresponding stress variation of the total frictional contact system. (4). The proposed finite element algorithm can capture the key phenomena of stress evolution in such a frictional contact system easily and will be applied to the further complicated interacting fault system in the earthquake research.

\section{References}

1. Xing H.L. and Makinouchi A.: A node-to-point contact element strategy and its applications. RIKEN Review: High Performance Computing in RIKEN 2000; 30:35-39

2. Xing H.L. and Makinouchi A.: Finite element analysis of a sandwich friction experiment model of rocks, PAGEOPH 2002; 159:1985-2009

3. Xing H.L. and Makinouchi A.: Finite element modeling of multiboby contact and its application to active faults, Concurrency and Computation: Practice and Experience 2002; $14: 431-450$

4. Xing H.L. and Makinouchi A.: Three dimensional finite element modeling of thermomechanical frictional contact between finite deformation bodies using R-minimum strategy, Computer Methods in Applied Mechanics and Engineering 2002; 191:4193-4214.

5. Ruina A.L.: Slip instability and state variable friction laws, J. Geophys. Res. 1983; 88: 10359-10370 